\title{
DÜBLIN
}

Technological University Dublin

ARROW@TU Dublin

2008-05-01

\section{Recent Applications of Chemical Imaging to Pharmaceutical Process Monitoring and Quality Control}

\author{
A. A. Gowen \\ University College Dublin \\ Colm O'Donnell \\ Technological University Dublin \\ Patrick Cullen \\ Technological University Dublin, pj.cullen@tudublin.ie
}

See next page for additional authors

Follow this and additional works at: https://arrow.tudublin.ie/schfsehart

Part of the Food Science Commons, and the Microbiology Commons

\section{Recommended Citation}

Gowen, A.A. et al (2008) Recent applications of chemical imaging to pharmaceutical process monitoring and quality control. European Journal of Pharmaceutics and Biopharmaceutics, 2008, Vol. 69 (1), pp.10-22.

This Article is brought to you for free and open access by the School of Food Science and Environmental Health at ARROW@TU Dublin. It has been accepted for inclusion in Articles by an authorized administrator of ARROW@TU Dublin. For more information, please contact arrow.admin@tudublin.ie, aisling.coyne@tudublin.ie, gerard.connolly@tudublin.ie.

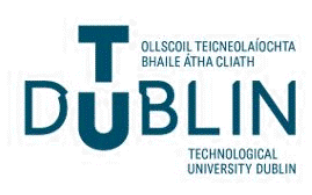




\section{Authors}

A. A. Gowen, Colm O'Donnell, Patrick Cullen, and S. Bell

This article is available at ARROW@TU Dublin: https://arrow.tudublin.ie/schfsehart/11 


\title{
Recent applications of Chemical Imaging to pharmaceutical process monitoring and quality control.
}

\section{A.A. Gowen ${ }^{\text {a* }}$, C.P. O'Donnell ${ }^{\mathrm{a}}$, P.J. Cullen ${ }^{\mathrm{b}}$, S.E.J. Bell ${ }^{\mathrm{c}}$.}

${ }^{a}$ College of Life Sciences, University College Dublin, Dublin 4, Ireland.

${ }^{\mathbf{b}}$ Department of Food and Pharmaceutical Science, Dublin Institute of Technology, Cathal Brugha Street, Dublin 1, Ireland.

'School of Chemistry and Chemical Engineering, Queen's University, Belfast BT9 5AG, Northern Ireland.

*corresponding author, email: aoife.gowen@ucd.ie

\begin{abstract}
Chemical Imaging (CI) is an emerging platform technology that integrates conventional imaging and spectroscopy to attain both spatial and spectral information from an object. Vibrational spectroscopic methods, such as Near Infra Red (NIR) and Raman spectroscopy, combined with imaging are particularly useful for analysis of biological/pharmaceutical forms. The rapid, non-destructive and non-invasive features of CI mark its potential suitability as a process analytical tool for the pharmaceutical industry, for both process monitoring and quality control in the many stages of drug production. This paper provides an overview of CI principles, instrumentation and analysis. Recent applications of Raman and NIR-CI to pharmaceutical quality and process control are presented; challenges facing CI implementation and likely future developments in the technology are also discussed.
\end{abstract}

Keywords: chemical imaging, non-destructive, pharmaceutical, drug, Raman, NIR 


\section{Table of Contents}

1. Introduction

2. Principles of Chemical Imaging

3. Chemical Imaging instrumentation

3.1 Pushbroom Acquisition

3.2 Staring Imager Configuration

4. Chemical Image analysis

4.1 Reflectance Calibration

4.2 Pre-processing

4.3 Classification

4.4 Image processing

5. Pharmaceutical Applications

5.1 NIR Chemical Imaging

5.1.1 Quality assessment

5.1.2 High throughput analysis

5.1.3 Coating Thickness

5.1.4 Compositional Information

5.1.5 Remote identification

5.1.6 Blend Uniformity

5.1.7 Process Related Information

5.1.8 Counterfeit Drug Identification

5.2 Raman Chemical Imaging

5.2.1 Minor component detection

5.2.2 Extraction of Process Related Effects

5.2.3 Particle size estimation 


\subsubsection{Tablet characterisation}

6. Challenges

6.1 Chemometrics

6.2 Raman Global Imaging

6.3 Image acquisition time

7. Future prospectives

8. Conclusions 


\section{Introduction}

The US Food and Drug Administration (FDA)-led process analytical technology (PAT) initiative is transforming approaches to quality assurance in the pharmaceutical industry. Core to the PAT initiative is increased process understanding by monitoring of critical performance attributes, leading to better process control and ultimately improved drug quality [1]. Potential advantages of PAT implementation include: reduced production cycle times, prevention of rejects, reduction of human error and facilitation of continuous processing to improve efficiency. With adequate knowledge of the ingredient-process relationship, the FDA will allow a pharmaceutical company to adjust their manufacturing process to maintain a consistent output without notification to the FDA [2].The real time release paradigm assures that when the last manufacturing step is passed, all final release criteria are met [3]. Adoption of such a quality by design approach would allow significant benefits to be gained by pharmaceutical companies: labour and time intensive finished product testing would be avoided if the product could be released based on in-process data. This possibility is driving pharmaceutical companies to gain a more complete understanding of their processes and input variable relationships to realise the benefits of PAT registrations.

Process monitoring and control are necessary at all stages of pharmaceutical processing, from raw material to packaged product characterisation. Traditional quality control methods such as High Performance Liquid Chromatography (HPLC) and Mass Spectroscopy (MS) are time consuming, destructive, expensive, require lengthy sample preparation and give no information about the distribution of components within a sample. Due to the destructive and time consuming nature of these methods, only small samples of drugs may be tested from given production 
batches [4]. Motivated by the PAT initiative, pharmaceutical analytical techniques are being developed to provide real-time process control to improve understanding of the chemical and physical processes that occur during pharmaceutical unit operations. Vibrational spectroscopic techniques such as Near infrared spectroscopy (NIRS) and Raman spectroscopy (RS) have emerged as valuable tools for pharmaceutical quality analysis.

NIRS is widely recognised as a valuable tool in pharmaceutical analysis for raw material testing, quality control and process monitoring, mainly due the advantages it allows over traditional methods, e.g. speed, little/no sample preparation, capacity for remote measurements (using fibre optic probes), prediction of chemical and physical properties from a single spectrum [5] and [6]. Räsänen and Sandler [7] provide a comprehensive review on the applications of NIRS in the development of solid dosage forms, documenting the usefulness of this technique for each of the manufacturing steps, from raw material screening to assessment of the final dosage form. Reich [5] reviewed the regulatory status of NIR analysis in the pharmaceutical industry, noting that although many pharmaceutical companies have successfully implemented NIR systems in QC labs for raw material identification and qualification, only a few quantitative NIR methods have yet gained regulatory approval.

Raman spectroscopy (RS) is based on the Raman effect: a substance, when irradiated with monochromatic light, scatters most of it at the incident frequency, but a small proportion of photons $(0.0001 \%)$ are scattered with shifted frequency; the inelastic scattering of a photon which creates or annihilates an optical phonon is known as the 
Raman effect. RS is highly selective; Raman spectra are more distinct and less overlapped than NIR spectra. Low signal to noise ratio is, however, a problem for RS, which arises due to the very small proportion of scattered photons that make up the Raman signal, and the need to use filters to remove the excitation line from the collected radiation [8]. The high cost of instrumentation has acted as a barrier to its routine industrial utilisation, nonetheless, the value of Raman spectroscopy in pharmaceutical analysis is well recognised; Rantanen [8] recently published a review documenting the process analytical applications of RS in the area of pharmaceutical dosage forms. Its potential application in dosage formulation, from chemical synthesis to tablet coating were described, along with a discussion of the main challenges facing its implementation in process analysis, which include: interfacing with process environment, sample heating during measurement and small sampling area.

Spectrometers integrate spatial information to give an average spectrum for each sample studied. The inability of NIR and Raman spectrometers to capture internal constituent gradients within samples may lead to discrepancies between predicted and measured composition. Furthermore, point-source spectroscopic assessments do not provide information on spatial distribution of different constituents, which is important in many pharmaceutical applications such as blending.

Chemical Imaging (CI) is an emerging technique that integrates conventional imaging and spectroscopy to attain both spatial and spectral information from an object. The terms Spectral and Chemical are often interchanged to describe this type of imaging: Spectral Imaging is an umbrella term that can be applied to almost any optical spectroscopic technique (e.g. IR, Raman, Fluorescence, UV), while Chemical Imaging 
refers to the vibrational spectroscopies (IR, NIR, Raman). By combining the chemical selectivity of vibrational spectroscopy with the power of image visualisation, CI enables a more complete description of ingredient concentration and distribution in inhomogenous solids, semi-solids, powders, suspensions and liquids. Another major advantage of $\mathrm{CI}$ is that, unlike traditional spectroscopic methods, separate classification standards are not required. The large number of individual spectra acquired across the spatial dimension of heterogeneous compounds provides a basis from which relative concentrations can be determined for each spatial location. Some features of CI are compared with NIRS and RS in Table 1.

One of the earliest papers concerning CI [9] reported its application for identification of chemical constituents of wheat. Technology has advanced since then; CI can now be implemented on the processing line. Industrial applications of NIR-CI include inline sorting of paper [10], real time quality control during andalusite production [11] and on-line classification of synthetic polymers [12]. There have been many publications documenting the utilisation of CI in medical diagnostics [13], [14] and [15]. However, it is still emerging as a tool for analysis of pharmaceutical processes and formulations. This review is structured to provide an overview of the principles, instrumentation and analytical techniques involved in Chemical Imaging, followed by a review of the recent applications of NIR-CI and Raman-CI to pharmaceutical process monitoring and quality control. Current challenges facing CI and likely future developments in the technology are also presented.

\section{Principles of Chemical Imaging}


Chemical Images are made up of hundreds of contiguous wavebands for each spatial position of a target studied. Consequently, each pixel in a Chemical Image contains the spectrum of that specific position. The resulting spectrum acts like a fingerprint, which can be used to characterise the composition of that particular pixel. Chemical Images, known as hypercubes, are three dimensional blocks of data, comprising of two spatial and one wavelength dimension, as illustrated in Figure 1. The hypercube allows for the visualization of biochemical constituents of a sample, separated into particular areas of the image, since regions of a sample with similar spectral properties have similar chemical composition.

It is currently unfeasible to obtain information in all three dimensions of a hypercube simultaneously; one is limited to obtaining two dimensions at a time, then creating a three dimensional image by stacking the 2 dimensional 'slices' in sequence. There are two conventional ways to construct a hypercube. One method involves acquisition of simultaneous spectral measurements from a series of adjacent spatial positions - the object is moved underneath an imaging spectrograph in what is known as pushbroom acquisition [16]. This method has been applied for hypercube acquisition in Raman mapping investigations [17] and [18]. Another method involves keeping the image field of view fixed, and obtaining images one wavelength after another in what is known as a staring imager configuration [5]. This method has been popular for construction of NIR-CI hypercubes in pharmaceutical applications [19] and [20].

\section{Chemical Imaging instrumentation}

Instrumentation for acquisition of Chemical Images varies from lab to lab: some research facilities have built their own instruments; others have purchased 
commercially available complete systems. The two main types of Chemical Imaging system are discussed here, namely staring imager and pushbroom configurations. Both configurations are applicable to both Raman and NIR imaging; although, as previously mentioned, the staring imager configuration is commonly applied to NIRCI investigations, while the pushbroom system is more typically applied to Raman mapping experiments. While the components of these systems vary, they can be schematically represented as in Figure 2. The following general components are usually required: illumination source, imaging optic, spectral encoder for wavelength selection, detector and acquisition system (usually a frame-grabber board interfaced with a PC). Advances in the production of low cost array detectors have meant faster data collection and cheaper Chemical Imaging systems; detection time depends on spatial and spectral resolution required, increasing with increasing resolution.

\subsection{Pushbroom Acquisition}

Pushbroom imaging systems require a translation stage for sample movement. Some Raman mapping instruments produce Chemical Images using an optical microscope coupled to a spectrometer [21]. These instruments are based on step and acquire acquisition mode: spectra are obtained at single points on a sample, then the sample is moved and another spectrum taken. This process is repeated until spectra of points spanning the entire sample are obtained. Advances in detector technology have reduced the time required to acquire Chemical Images. Raman line mapping experiments are also possible [22], where the spectrum of each pixel in a line of sample is simultaneously recorded by an array detector. In Raman mapping the sample is usually illuminated by a laser source in the visible or NIR wavelength range. A line of light reflected from the sample enters the imaging optic (usually a 
microscope lens) and is separated into its component wavelengths by diffraction optics contained in the spectral encoder, which is usually a spectrometer in point mapping systems and a spectrograph in line mapping systems; a two-dimensional image (spatial dimension $\mathrm{x}$ wavelength dimension) is then formed on the detector. The sample is moved past the objective lens on a motorized stage and point or line images acquired at adjacent points on the object are stored on a PC for further analysis. Charge Coupled Device (CCD) detectors (sensitive between 400-1000 nm) are typically used as detectors in Raman imaging, while Fourier Transform (FT) Raman imaging systems require more expensive longer-wavelength focal-plane array (FPA) detectors [21].

\subsection{Staring Imager Configuration}

The major advantage of this method over the pushbroom technique is that it doesn't require any moving parts.The sample is usually illuminated with a diffuse source of NIR light (for NIR-CI) or dilated laser source (for global Raman Imaging). Light reflected from the sample is captured via an imaging optic; for microscopic images a microscope objective lens is used. The light is then passed through a filter, which acts as a spectral encoder in this arrangement, separating images into their spectral components. Early staring configuration Chemical Images were obtained using a series of bandpass filters selective to certain wavelengths of light, which were often mounted on a wheel for ease of switching. Recent advances in tunable filters, which can select wavelengths rapidly through software control, have rendered the time consuming bandpass method largely obsolete. Acousto-optic Tuneable Filters (AOTFs) and Liquid Crystal Tuneable Filters (LCTFs) are the two most widely used tuneable filters in CI. AOTFs have been used in the construction of commercially 
available NIR-CI systems [23], while LCTFs show greater promise for filtering of Raman images [21]. After spectral encoding, an image of the sample at the selected wavelength is recorded on a detector, which is usually a CCD detector for Raman Imaging and an FPA for NIR Imaging. Images at subsequent wavelengths are stacked to form a hypercube and stored on a PC for further analysis.

\section{Chemical Image analysis}

Chemical Image files are typically immense; for example, a hypercube with spatial dimensions of $256 \times 256$ pixels operating at 150 wavebands contains more than 65,000 spectra, each with 150 data points. In attempting to extract useful information from such information-dense datasets a multidisciplinary approach is required, involving advanced image processing and multivariate statistical methods. Numerous techniques exist to mine the chemical, physical and spatial information hidden in Chemical Images. The challenge is to reduce the dimensionality of the data while retaining important spectral information with the power to classify important areas of a sample effectively. Typical steps involved in analysing Chemical Images are outlined in Figure 3 and described below.

\subsection{Reflectance Calibration}

This is carried out to account for the background spectral response of the instrument and the 'dark' camera response. For reflectance measurements, the background is obtained by collecting a hypercube from a uniform, high reflectance standard or white ceramic; the dark response is acquired by turning off the light source, completely covering the lens with its cap and recording the camera response. The corrected reflectance value $(\mathrm{R})$ is calculated as follows: 
$\mathrm{R}=($ sample - dark $) /($ background-dark $)$

\subsection{Pre-processing}

Pre-processing is usually performed to remove non-chemical biases from the spectral information (e.g. scattering effects due to surface inhomogeneities, interference from external light sources, random noise) and prepare the data for further processing. Mathematical treatments to compensate for scatter-induced baseline offsets include multiplicative scatter correction, Savitzky-Golay derivative conversion and standard normal variate correction. Normalisation algorithms can be used to compensate for baseline shifts and intensity variations resulting from path length differences. Other operations usually carried out at the pre-processing stage include thresholding and masking to remove redundant background information from the hypercube.

\subsection{Classification}

Hypercube classification enables the identification of regions with similar spectral characteristics, providing information on the physical and chemical properties of a sample, their distribution and concentration. Due to the large size of hypercubes (which can exceed $50 \mathrm{MB}$, depending on image resolution, spectral resolution and pixel binning) complex multivariate analytical tools are usually employed for classification. Analysis of each spectrum in a hypercube can be performed with chemometric tools designed for bi-linearly structured data sets. In order to apply conventional chemometric techniques in Chemical Image analysis, it is necessary to restructure the hypercube: in a process known as spectral unfolding the 3-dimensional hypercube is rearranged into a 2-dimensional matrix, by appending the two spatial dimensions. After classification, the image matrix is folded back into 3-dimensional 
form from which Chemical Images are produced.Unsupervised classification methods, such as principal component analysis (PCA), k-means clustering and fuzzy clustering, require no apriori information about the dataset, and are useful as exploratory tools to extract important features. Supervised techniques, such as partial least squares (PLS), linear discriminant analysis (LDA), Fishers discriminant analysis (FDA) and artificial neural networks (ANN), require prior knowledge about the data and selection of pre-defined training sets against which to classify the data. Training sets can be selected from regions of the hypercube using previous knowledge of sample composition. Another approach involves selection of training sets from areas identified by the unsupervised analyses as containing a specific component; combining unsupervised and supervised methods in this way provides a means of locating constituent components without apriori knowledge of the sample.

\subsection{Image processing}

Image processing is carried out to convert the contrast developed by the classification step into a picture depicting component distribution. Greyscale or colour mapping with intensity scaling is commonly used to display compositional contrast between pixels in an image. Single wavelength images are easy to produce but can be misleading, since the depicted images may be biased or dominated by variations in thickness across sample surface; multi-wavelength images give a better representation of the sample composition [24]. Image fusion, in which two or more images at different wavebands are combined to form a new image [25] is frequently implemented to provide even greater contrast between distinct regions of a sample. Images may be combined using algorithms based on straightforward mathematical operators, e.g. addition, subtraction, multiplication and division. One example is the 
band ratio method, in which an image at one waveband is divided by that at another wavelength [26] and [27]. Histograms are another tool, useful for depicting pixel distribution of components.

\section{Pharmaceutical Applications}

\subsection{NIR Chemical Imaging}

NIR Chemical Imaging (NIR-CI) has been applied to a number of process and quality related investigations for pharmaceutical products, including quality assessment, highthroughput analysis, coating thickness, compositional information, remote identification, blend homogeneity, extraction of process related effects and counterfeit drug identification. Table 2 presents a summary of the published applications of NIRCI to pharmaceutical analysis since 2001. The following sections describe each application in more detail.

\subsubsection{Quality assessment}

Westenberger et al. [30] compared quality assessment of pharmaceutical products purchased via the internet using a variety of traditional (HPLC-based) and nontraditional (NIR, NIR-CI) techniques. A staring imager chemical imaging system was utilised to obtain Chemical Images of individual specimens at 121 wavelengths between $1050-1700 \mathrm{~nm}$. The NIR-CI technique had an added advantage over traditional techniques in its ability to detect suspect manufacturing issues, such as blending and density pattern distribution of components within a drug product, allowing direct qualitative comparison with control products. It was also noted that the NIR and NIR-CI techniques are non-destructive so that defective samples could be scanned and saved for further testing. 
Another paper reports the utilisation of NIR-CI for qualitative analysis of pharmaceutical forms [20]. A microscope coupled with a FPA detector constituted the NIR Chemical Imaging system in this case. Peak wavelength height and Principal Component Analysis (PCA) were used for qualitative analysis of surface contamination on solid dosage forms and to identify dissolution properties of a number of samples. While NIR imaging alone was sufficient for describing dissolution problems, imaging combined with PCA was necessary for identifying contamination.

\subsubsection{High throughput analysis}

The huge volume of production encountered in the pharmaceutical industry necessitates high throughput quality analysis techniques. NIR-CI may be adapted to perform analysis on multiple samples in a single field of view by increasing the distance between the camera and target. Fast and nondestructive identification of active ingredients and excipients in whole tablets can be achieved, even through packaging [38] and [39]. A paper published in 2001 [40] reported the use of multispectral imaging for simultaneous identification and composition of multiple individual tablets in blister packaging. A FPA interfaced with a tuneable interference filter was used to obtain images of 1286 tablets in blister packs at wavelengths in the range $1678-2312 \mathrm{~nm}$. It was stated that multispectral imaging offers a potential speed advantage of approximately 30,000 over HPLC when determining moisture and salicylic acid content in single packaged aspirin tablets, and that NIR-CI is up to 1000 times faster than NIRS of single tablets. Hamilton \& Lodder [38] reported that a typical NIR chemical imaging system could simultaneously analyse around 1300 
tablets in blister packaging. A more recent paper [39] reported high throughput analysis of pharmaceutical tablet content uniformity using NIR-CI. A large field of view NIR-CI device was used to take simultaneous Chemical Images of 20 tablet samples at 61 wavelengths. The total time for image acquisition was less than 2 min, while the conventional UV method took approximately half a working day for sample analysis. It was observed that the mean intensity of images at $1600 \mathrm{~nm}$ was proportional to active pharmaceutical ingredient (API) concentration; the authors suggested that a multispectral instrument based on a small number of wavelengths around $1600 \mathrm{~nm}$ could be built to rapidly identify API concentration of these tablets in packaged form.

\subsubsection{Coating Thickness}

Lewis, Kidder \& Lee [35] applied NIR-CI for quantification of coating thickness in a single time release micro-sphere. NIR Chemical Images demonstrated the differences in chemical structure of the tablet core and coating, enabling quantitative information on coating thickness and homogeneity to be determined. Uneven coating could be clearly identified by visualisation of the total intensity image at $2080 \mathrm{~nm}$. A group of 135 microspheres containing two different microsphere types was also imaged in this study. Discrimination of microsphere type was readily visualised in a spectral difference image.

\subsubsection{Compositional Information}

Many new drug release systems require complex tablet architecture. In 2001, Lewis, Carroll \& Clark published a paper [28] showing how NIR-CI could be employed for qualitative description of composition and architecture of a solid dosage form. A time- 
release granule was bisected and an NIR-CI system was used to acquire Chemical Images at 71 wavelengths from 1000 to $1700 \mathrm{~nm}$ in approximately $2 \mathrm{~min}$. Unsupervised PCA was used to identify spatial and chemical variance in the sample. Loading scores of the principal components were used to produce score images of the sample which clearly showed boundaries in the sample where chemical composition varied.

\subsubsection{Remote identification}

Hamilton \& Lodder [38] investigated the potential implementation of a far field imaging system to identify pharmaceutical capsules. Individual gelatin capsules, subjected to degradation by exposure to formaldehyde, were imaged from a distance of $500 \mathrm{~m}$ using a telescopic lens interfaced with a NIR imaging system. Images were only collected at 6 wavelengths to reduce data collection time. Principal component regression of the spectra against time of exposure to formaldehyde gave promising results $\left(r^{2}=0.89, \mathrm{SEP}=1.17 \mathrm{~h}\right)$. Similar results were published in another paper [41].

\subsubsection{Blend Uniformity}

Powder blend homogeneity in final dosage product poses a significant problem in pharmaceutical quality assurance; poor blending tends to adversely affect tablet hardness, appearance and dissolution kinetics. Lyon et al. published a paper in 2002 [33] comparing the ability of NIR-CI and NIRS in distinguishing blending grade for a selection of final dosage forms. Individual tablets with different blending grades were imaged at 71 wavelengths from 1000-1700 nm using a staring imager configuration Chemical Imaging system. PLS was employed for hypercube analysis. While NIRS was limited in its ability to evaluate drug product homogeneity, NIR-CI provided the 
opportunity to investigate localised micro-domains of ingredients within a drug. It was noted that physical and chemical abnormalities that made minimal contribution to the bulk tablet would be undetected by traditional NIRS but detected by NIR-CI.

More recently, the US FDA has embarked on an industrial collaborative project which aims to develop Chemical Imaging instrumentation for blend process monitoring [42]. A prototype instrument, the Blend Monitor (see schematic in Fig. 4) has been designed for application of NIR Chemical Imaging at a process scale. This instrument is based on a LCTF staring imager configuration; a fibre optic bundle is interfaced with the blender to collect and transmit NIR spectral information from the blend to the NIR imaging spectrometer [34]. The instrument thus records Chemical Images of samples inside the blender. However, rotation of the blender is problematic as the Blend Monitor cannot co-rotate, requiring the blender to be stopped before measurement. Therefore, this instrument cannot currently be used "in process" for online measurements.

\subsubsection{Process Related Information}

Pharmaceutical products undergo various chemical and physical changes during processing. Characterising the nature and sources of these changes is a major challenge for PAT. Clarke [31] reported the utilisation of NIR-CI microscopy for identification of tablets which experienced problems during processing and for investigation of pre-tabletting blends with dissolution issues. Chemical Images of samples were obtained at 121 wavelengths between $1100-1700 \mathrm{~nm}$. PCA was performed on the Chemical Images of tablets which experienced problems during processing; image reconstruction was achieved using PCA scores and loadings. PLS 
classification was used for the pre-tabletting blends with dissolution issues; cluster size of ingredients was measured to investigate the effect of compaction force on dissolution properties. In a similar study, the potential of NIR-CI for investigating the effects of processing on tablet dissolution was examined [32]. It was shown that NIRCI could be used for the prediction of coating time and to understand batch differences due to different processing conditions.

\subsubsection{Counterfeit Drug Identification}

The problem of counterfeit drugs is widespread; so-called "high-quality" counterfeit drugs are difficult to detect. Rodionova et al. [36] reported advances in the feasibility of detecting counterfeit drugs using both NIRS and NIR-CI. Chemical Images were obtained for two tablet powder samples at 128 wavelengths in the range $900-1700$ nm. PCA was applied for image analysis, and clustering evident in the score plot of the first and third principal components was related to drug composition. The authors remarked that NIR-CI is useful for identifying small differences in ingredient distributions, which is important for identification of so-called "high quality" counterfeit samples. A similar study reported the use of NIR-CI in the analysis of multiple samples to discriminate between counterfeit and non-counterfeit tablets without prior knowledge of sample architecture or composition [37].

\subsection{Raman Chemical Imaging}

Raman Chemical Imaging has been applied to many diverse fields, including polymer classification [43], dental sample characterisation [44], process monitoring of semiconductors [45] and detection of filtered waterborne bacteria [46]. A large number of patents for Raman Imaging applications exist, including one describing the 
application of Raman imaging microscopy for the evaluatation of drug action within living cells [47]. In most Raman Imaging experiments, a microscope is attached to the imaging system to enable molecular characterisation. Some recently published applications of Raman spectroscopy in pharmaceutical analysis, summarised in Table 3, include minor component detection, detection of process related effects, particle size estimation and tablet characterisation. These applications are discussed in more detail in the following sections.

In simple cases Raman imaging can provide data on API distribution within tablets in a very straightforward fashion. For example, Figure 5 shows a composite Raman image comparing data from two tablets (half of each is shown to highlight the differences) with the same overall composition but different formulation protocols. These pushbroom images were recorded at $100 \mu \mathrm{m}$ micron spacing on a 70/70 grid i.e. a total scan area $7 \mathrm{~mm}$ square to allow the entire face of each tablet to be imaged. Accumulation time was $5 \mathrm{~s}$ per point, leading to a total acquisition time of approximately $7 \mathrm{~h}$. Both tablets were prepared from mefenamic acid (API) (20\% by mass) with Lutrol binder and lactose bulking agent. In one case the constituents were prepared by normal co-melt fluidised bed granulation i.e. the three constituents were granulated in a fluidised bed whose temperature was taken above that of the melting point of the binder. The solubility of this API in the Lutrol binder is relatively low, which leads to non-uniform distribution in the granules and ultimately the tablets prepared from them, as shown in the Figure. The corresponding image with higher uniformity of colour is one where the API was first dissolved in binder and the resulting mass reground before being granulated in a fluidised bed as before. This procedure improves the uniformity of API distribution in the granules and the tablets 
prepared from them. There are numerous ways to treat these data, such as plotting the relative band intensities of the API and binder/excipient. However, in these images the data at each pixel were compared to a standard (average) spectrum of a sample which had API and binder/excipient band intensities at the desired level by calculating a simple correlation coefficient. In these images the positions where the correlation was high are white/pink while positions where there is poorer correlation are green/blue. The spectra at the areas showing blue are essentially pure API, as evidenced by comparison of spectra obtained at these positions with that of a pure API standard.

\subsubsection{Minor component detection}

Many new drugs contain small concentrations of potent APIs delivered by complex delivery systems. It is imperative that content uniformity can be maintained in such systems. Šašic [49] published a study on Raman Chemical Imaging of low content API pharmaceutical formulations in which Raman maps were obtained from the flattened surfaces of 6 tablets using a pushbroom Raman Imaging system. Univariate images, produced from the intensity recorded at each pixel at individual wavelengths were difficult to interpret, and the author reported that reliable API Raman signal was only detected upon applying Principal Component Analysis (PCA) to the data.

Šašic \& Clark [22] investigated the performance of Raman line-mapping compared with global illumination Raman systems for the analysis of tablets and formulation blends. A pushbroom configuration was used in the line-mapping experiment, while a staring imager configuration was used in the global experiment. Raman line-mapping technology was demonstrated as preferable for identifying minor components in 
samples, whereas the global illumination system was recommended for imaging of spatially isolated strong Raman scatterers. These researchers also reported a novel chemometric technique for analysis of Raman Images of pharmaceutical samples [53]. This method, called sample-sample two dimensional correlation, involves analysis of the covariance matrix of the Raman mapping spectral matrix. The most unique spectra in the mapping matrix are identified by analysing the slices of the covariance matrix at positions where the covariance values are maximum; Chemical Images are then produced by visual selection of the wavenumbers in the extracted spectra that are least overlapped. The authors demonstrated that this method was suitable for producing reliable images of unknown samples of pharmaceutical tablets containing up to 4 active components.

\subsubsection{Extraction of Process Related Effects}

Henson \& Zhang [18] published an article on drug characterization of low dosage pharmaceutical tablets using Raman microscopic mapping. Raman Images were obtained using a Raman line-mapping instrument. Domain size and spatial distribution of the API and major excipients were estimated from Raman maps of the tablets. It was demonstrated that domain size of the API was dependent upon the particle size distribution of the ingoing API material; therefore, the Raman maps could be used to indicate the source of API used in tablet manufacturing. The potential utility of Raman microscopic mapping in manufacturing process optimization or predictive stability assessments was also outlined.

\subsubsection{Particle size estimation}


Doub et al. [48] reported the application of Raman-CI for ingredient specific particle size characterisation of nasal spray formulations. Samples of aqueous suspension were sprayed onto inverted aluminium-coated glass microscope slides and allowed to dry before imaging. A Raman Imaging particle size distribution (PSD) protocol was developed and validated using polystyrene (PS) miscrosphere size standards. Good statistical agreement was obtained between reported PS particle sizes and those estimated using Raman-CI. Moreover, Raman-CI was suitable for distinguishing between active samples and placebos. It was noted, however, that vigorous validation of Raman-CI for PSD is required, due to the high variability associated with replicate measurements.

\subsubsection{Tablet characterisation}

Šašic [29] published findings from a study in which Raman-CI was compared with NIR-CI for determining spatial distribution of multiple components in tablets. Multivariate Raman images could identify all of the components in each tablet studied, while only two or three components could be imaged using NIR imaging. It was demonstrated that the eigenvalue-based criteria for choosing principal components was ineffective for these Raman Chemical Images, and the author remarked that the basic postulates of PCA could pose a significant problem when applied to Raman mapping spectra. For NIR mapping spectra, interpretation of PCA proved difficult; problems were associated with associating pure component spectra and loadings. It was demonstrated that PCA was less efficient for producing NIR-CIs than for Raman CIs. 
Raman Chemical Imaging in conjunction with multivariate statistical analysis enables the extraction of information about the concentration and distribution of pure compounds in a sample. A paper published in 2004 [52] elucidated a multivariate approach to both global and local Chemical Imaging and tested the proposed methodology on Raman images of tablets. Five tablets, each with varying amounts of API and excipient, were imaged using a Raman point mapping system with 45 x 45 grid points and 576 wavelengths between 8525-16420 nm. Global Principal Component Analysis (PCA) was applied to the entire data set to determine the number of compounds present. This was followed by local PCA, applied sequentially to small subsets of the data, to examine the distribution of compounds in the individual tablet samples. An interative optimisation approach was employed to minimise model error.

Zhang, Henson and Sekulic [54] reported on the merits and drawbacks of applying a selection of multivariate data analysis techniques (PCA, cluster analysis, direct classical least squares (DCLS) and multivariate curve resolution (MCR)) for analysing Raman images of a model pharmaceutical tablet. Raman maps were obtained using a microscopic Raman line-mapping instrument. It was noted that, although PCA was effective for exploratory data analysis, there was generally no oneto-one correspondence between PCs and a chemical components. The potential use of cluster analysis as a tool for automatic segmentation of images enabling visualisation of distinct regions with similar chemical composition in drugs was also described; this method would be particularly valuable for characterisation of solid dosage with distinct spatial distribution character, such as controlled release formulations. 
Bell et al. [51] , [55], [56] and [57] published findings on the development of quantitative analytical methods for Raman analysis of solid dosage forms. In one paper, this research team reported results on Raman mapping of seized ecstasy tablets [51]. Raman point mapping was performed on tablet samples on both microscopic and macroscopic scales to investigate the effects of sampling size on measurement error. Micro-Raman mapping was achieved using a Raman microscope with a motorised $x-y$ translation stage, and macro-Raman maps were obtained using a Raman spectrometer with an $x-y-z$ stage. Figure 6 shows a coarse Raman map of the peak area of the strongest MDMA band in the Raman spectra of a seized ecstasy tablet, taken at 785 nm with a Raman microscope ( 8 x 8 grid, $10 \mu \mathrm{m}$ spacing, 50x objective lens). Although only a small number of grid points were measured (to decrease time and cost of analysis), inhomogeneous distribution of tablet components (MDMA, caffeine and lactose) is evident. It was demonstrated that acceptably low sampling errors could be achieved when less than 100 points were sampled, and that sampling error could be estimated by comparison of sub-sets of data in the grid. It was reported that for the macro-Raman system, grid acquisition time of $1 \mathrm{~min}$ for individual tablets could potentially be realised.

Another more recently published article discusses the impact of spatial sampling density in Raman Imaging [50]. In this investigation, Raman point mapping was applied to a fixed (magnified) area of an over-the-counter drug using spatial steps ranging from 10 to $100 \mu \mathrm{m}$. The coarse grid (with $100 \mu \mathrm{m}$ steps) Raman image took a few minutes to acquire, while the fine grid (with $10 \mu \mathrm{m}$ steps) took several hours to record. It was demonstrated that the coarse measurement could be useful for selection of regions which required further fine measurement. Although the fine grid method is 
time consuming, it would allow for visualisation of the chemical distribution of the sample with details of crystal morphology.

\section{Challenges}

The potential applications of Chemical Imaging for pharmaceutical process monitoring and quality analysis have been highlighted in this article. Nevertheless, it is worthwhile to note that Chemical Imaging is not an end-all solution for pharmaceutical analysis. For many applications, single point spectroscopic methods are sufficient (e.g. for bulk sample identification or quantitative determination of the average composition of a substance). On the other hand, there remain key areas in pharmaceutical process monitoring for which CI is quite possibly the best solution, i.e. measuring spatial heterogeneity, component distribution, coating thickness, particle size, contaminant detection and high throughput analysis. However, the technology is not without its limitations; some challenges face the routine implementation of CI in the pharmaceutical industry and are explored below.

\subsection{Chemometrics}

Šašic [29] reported that using PCA on both Raman and NIR spectra of pharmaceutical tablets led to problems associated with associating pure component spectra and loadings. Some authors have remarked that conventional chemometric methods such as PCA and PLS may not be suitable for analysing Chemical Images, since these techniques were developed for analysing single spectra [58] and [59]. To overcome the limitations of conventional chemometric approaches, a number of novel chemometric methods have been proposed. One example is support vector machines (SVMs). SVMs belong to the group of machine learning algorithms that use 
optimization tools, which work to identify the optimal hyperplane as a decision surface to discriminate between classes of interest. These chemometric tools are rapidly emerging as superior tools for a diverse group of pattern recognition and classification applications [60] and [61]. The application of novel chemometric techniques such as SVMs in CI for pharmaceutical analysis may result in improved detection capability.

\subsection{Raman Global Imaging}

In the majority of published research on Raman Imaging (Table 3), hypercubes are acquired by means of point or line mapping. Šašic [29] stated that "line-mapping systems are more widely understood and are more frequently used in practise". However, Schlucker et al. [62] reported that higher spatial resolution is obtained from global Raman Imaging than point or map Raman Imaging, and this enables subtle morphological features on test samples to be imaged. Moreover, Raman line and point mapping experiments can be painfully time consuming (up to $20 \mathrm{~h}$ ). Raman global imaging experiences difficulties when a large field of view is required, since it is difficult to flood these areas with laser power [63]. Another problem that may arise from the wide field illumination required in global Raman imaging is spectral averaging due to photon migration [28]. It is clear that these challenges need to be overcome in order to realise the full benefits of global Raman Imaging as a PAT tool.

\subsection{Image acquisition time}

A major factor limiting the implementation of CI for routine process monitoring arises from the relatively lengthy times necessary for hypercube image acquisition processing and classification [64]. Depending on target size, image and spectral 
resolution required, acquisition time can range from 2 - 4 min (some Raman mapping experiments require up to $20 \mathrm{~h}$ acquisition time), while processing and classification time are largely dependant on computer hardware and software capabilities. However, it can be expected that future developments in the technology will shorten processing and acquisition time, enabling real-time CI process monitoring systems. Chemical Image Fusion, discussed in the next section, is a promising technique for reducing processing time in $\mathrm{CI}$.

\section{Future prospectives}

Future improvements in precision and speed in Chemical Imaging are likely to arise with increased computer processing speeds, improved cameras, faster hardware, more accurate and efficient algorithms. Further applications of this emerging technique to pharmaceutical product quality and process monitoring will certainly arise with the development novel solutions for online data acquisition and more powerful data evaluation strategies. A selection of novel technologies related to Chemical Imaging are discussed in the following sections.

Chemical Image Fusion is a technique proposed by Clarke et al. [65] to exploit the complementary benefits of NIR and Raman microscopy. Raman and NIR Chemical Images from the same area of a target are acquired, giving rise to a combined Chemical Image that visually describes the entire formulation. In Clarke's study, combined images of pharmaceutical samples were obtained by merging Chemical Image maps of samples obtained from separate FT-NIR and Raman instruments. Reference marks were used to ensure that the same area was imaged on each instrument. Peak area measurements of baseline corrected single spectroscopic bands 
which were unique to the different sample components were used to generate images of each sample for particle size and distribution analysis. As instrumentation and chemometric methods to process this type of data are developed, it is likely that Chemical Image Fusion will find wider application in the field of pharmaceutical analysis

An exciting new technique, known as Integrated Chemical Imaging (ICI), has been proposed to reduce the data load and increase processing speed in Chemical Imaging [66]. Innovative spectrometer designs have been developed that speed up the process by enabling the sensing detector to do some of the computation. Once a set of training spectra at all available wavelengths has been accumulated, it is possible to rationally select molecular filter (MF) materials to perform PCA on the incoming data by weighting signals from spectral regions with most variability. Integration of such selective molecular filters (MF) with detectors would enable molecular computing to replace traditional chemometric methods, e.g. PCA. This is the same as physically implementing chemometric calculations in spectroscopic systems [67]. Rapid analysis coupled with reduced data storage requirements indicate that the practical realisation of ICI systems as PATs is not far away.

In a recent paper on future outlook for NIR-CI instrumentation, Bellon-Maurel [68] stated that imaging systems based on electronically tuneable filters (AOTF \& LCTF) rather than filter-based or pushbroom systems would become the preferred choice for Chemical Imaging. She cited the requirement of sample movement for pushbroom systems and the limited throughput and excessive time consumption of filter based systems as reasons for their diminished future use. On the other hand, it could be 
argued that with improvements in acquisition speed and spectral resolution, pushbroom systems may find a place for in process monitoring of pharmaceutical dosage forms on conveyor belt systems. At line pushbroom systems, in which tablets would be diverted onto a moving stage for Chemical Imaging, would facilitate analysis of a significantly higher number of tablets than traditional lab methods, albeit not the desired complete assessment. Bellon-Maurel predicts that the market for NIRCI instrumentation will increase in the coming years, especially in the pharmaceutical industry due to the FDA PAT initiative. She predicts that the price of imaging spectrometers will fall, opening their way into routine pharmaceutical analysis. The development of an average cost imager by combining microelectro-mechanical systems (electrically programmable diffraction gratings) with spectrometers would obviate the need for costly array detectors.

\section{Conclusions}

The integration of spectroscopic and digital imaging in Chemical Imaging (CI) produces position referenced spectra, which are valuable for the analysis of complex multi-constituent samples. A major advantage of CI for the pharmaceutical industry is its power to potentially assess all tablets in a production batch (not just a sample set), thereby significantly reducing variability and risk. Moreover, its ability to spatially localize drug constituents represents a vast improvement on average concentration methods such as HPLC and MS. The non-destructive, rugged and flexible nature of Chemical Imaging (CI) makes it an attractive PAT for identification of critical control parameters that impact on finished product quality. Judging by the continuing emphasis on process analytical technologies to provide accurate, rapid, nondestructive analysis of pharmaceutical processes it is likely that CI will be 
increasingly adopted for process monitoring and quality control in the pharmaceutical industry as has been the case with NIR spectroscopy. Future innovations in CI equipment manufacture are likely to depress purchase costs and encourage more widespread utilisation of this emerging technology in the pharmaceutical sector. 
Table 1. Comparison of NIR spectroscopy (NIRS), Raman spectroscopy (RS) and Chemical Imaging (CI).

\begin{tabular}{|l|l|l|l|}
\hline Feature & NIRS & $R S$ & $C I$ \\
\hline Spatial information & & & $\checkmark$ \\
\hline Spectral information & $\checkmark$ & $\checkmark$ & $\checkmark$ \\
\hline Multiconstituent information & $\checkmark$ & $\checkmark$ & $\checkmark$ \\
\hline Sensitivity to minor components & & & $\checkmark$ \\
\hline Tolerance to sample geometry & $\checkmark$ & & $\checkmark$ \\
\hline $\begin{array}{l}\text { Quantitative information without having } \\
\text { to run separate calibration samples }\end{array}$ & & & $\checkmark$ \\
\hline
\end{tabular}


Table 2. Recent pharmaceutical applications of NIR Chemical Imaging

\begin{tabular}{|l|l|l|}
\hline Configuration & Application & Reference \\
\hline \hline Pushbroom & Compositional information & {$[28],[29]$} \\
\hline Staring imager & Quality assessment & {$[20],[30]$} \\
\cline { 2 - 3 } & $\begin{array}{l}\text { Process related } \\
\text { information }\end{array}$ & {$[31],[32]$} \\
\cline { 2 - 3 } & Blend uniformity & {$[33],[34]$} \\
\cline { 2 - 3 } & Coating thickness & {$[35]$} \\
\cline { 2 - 3 } & Counterfeit drug detection & {$[36],[37]$} \\
\cline { 2 - 3 } & Remote identification & {$[38],[41]$} \\
\cline { 2 - 3 } & High throughput analysis & {$[38],[39]$} \\
\hline
\end{tabular}


Table 3. Recent pharmaceutical applications of Raman Chemical Imaging

\begin{tabular}{|l|l|l|}
\hline Configuration & Application & Reference \\
\hline Staring Imager & Particle size estimation & {$[48]$} \\
\hline $\begin{array}{l}\text { Pushbroom } \\
\text { \& } \\
\text { Staring Imager }\end{array}$ & $\begin{array}{l}\text { Minor component } \\
\text { detection }\end{array}$ & {$[22],[49]$} \\
\hline Pushbroom & Tablet characterisation & {$[17],[29],[50],[51],[52],[53],[54]$} \\
& $\begin{array}{l}\text { Extraction of Process } \\
\text { Related Effects }\end{array}$ & {$[18]$} \\
\hline
\end{tabular}




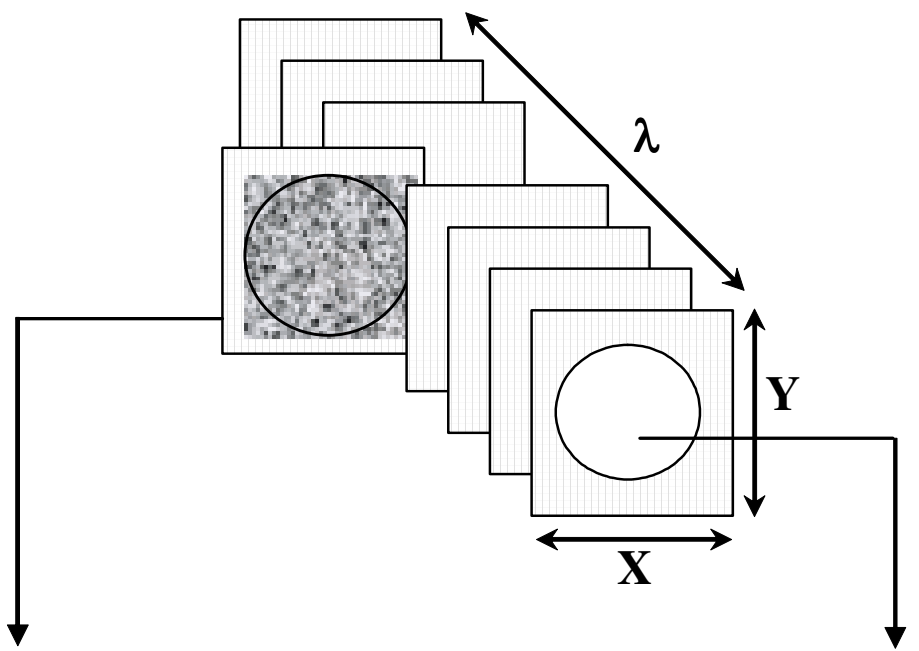

\section{Image plane at $\lambda_{i}$}

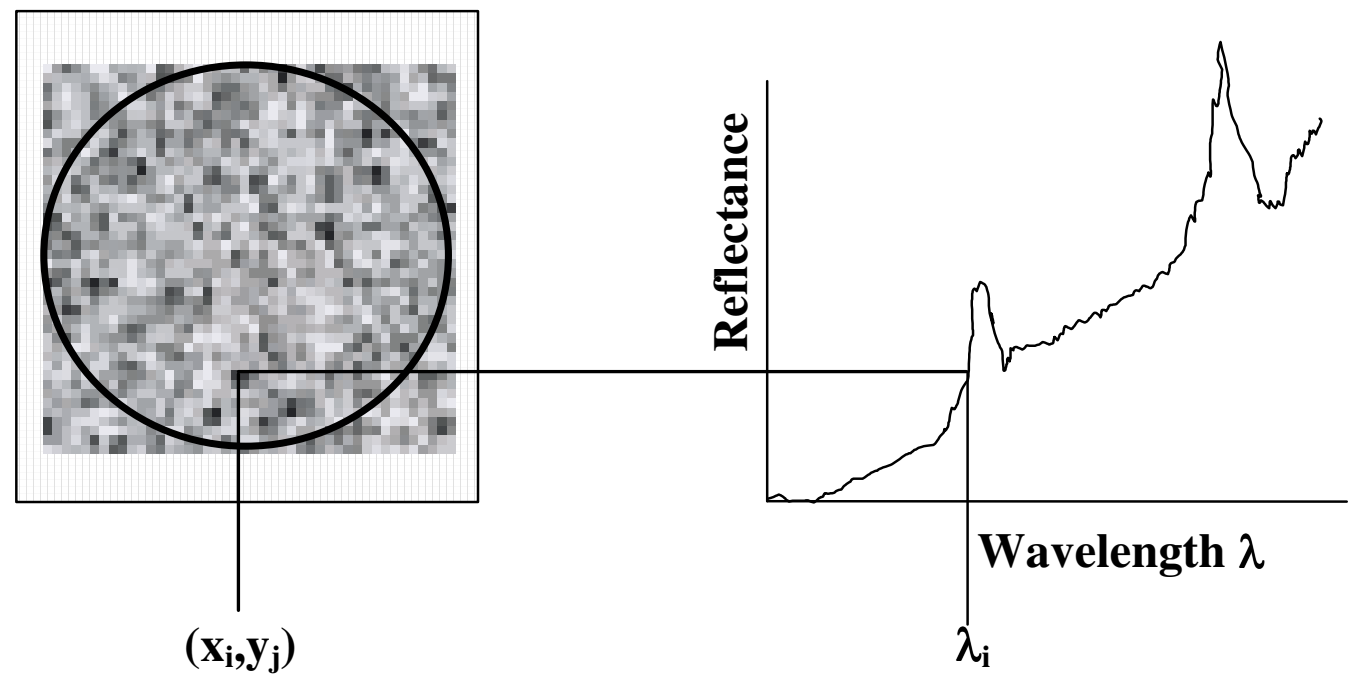

Fig. 1. Schematic representation of hyperspectral imaging hypercube showing the relationship between spectral $(\lambda)$ and spatial $(X, Y)$ dimensions. 


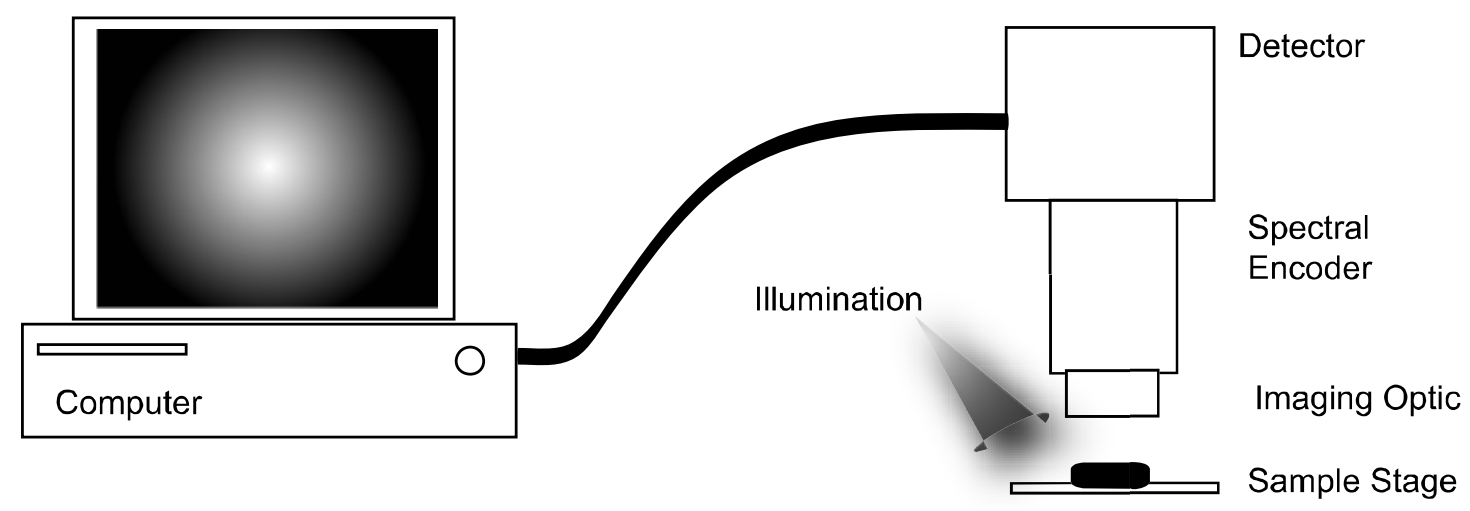

Fig. 2. Typical components of a Chemical Imaging system 


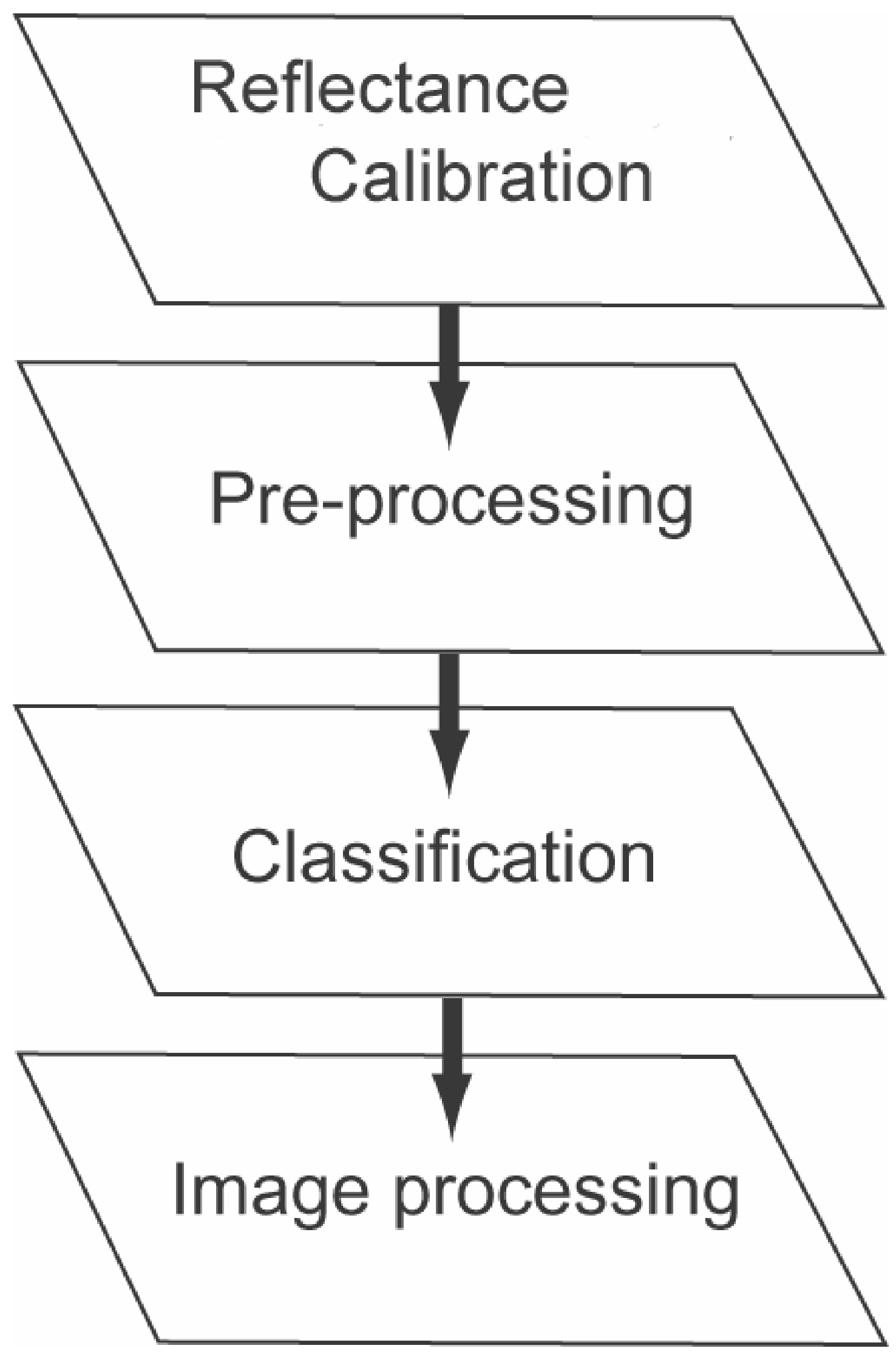

Fig. 3. Schematic diagram of Chemical Image data analysis process 


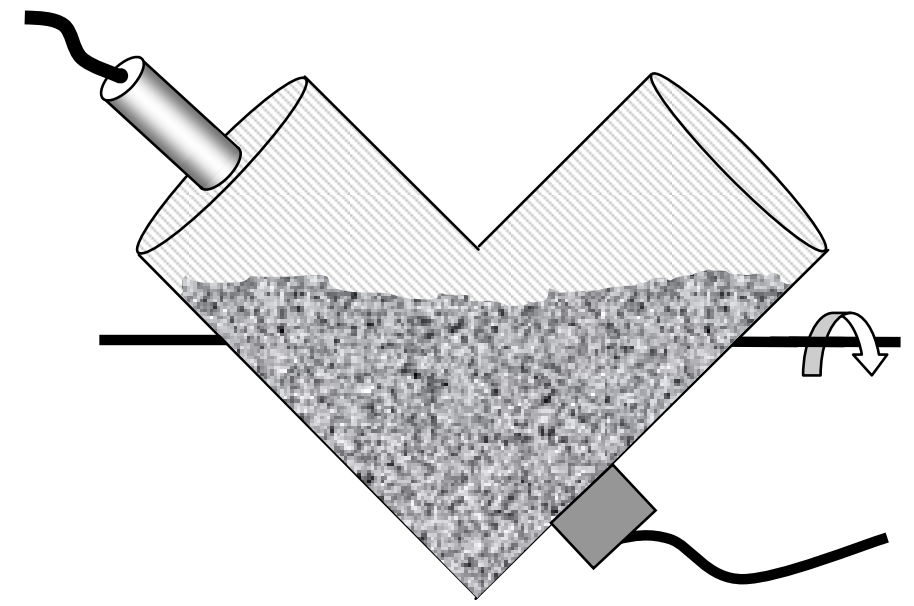

Figure 4. Schematic of NIR Chemical Imaging blend monitor mounted to a Vblender. 


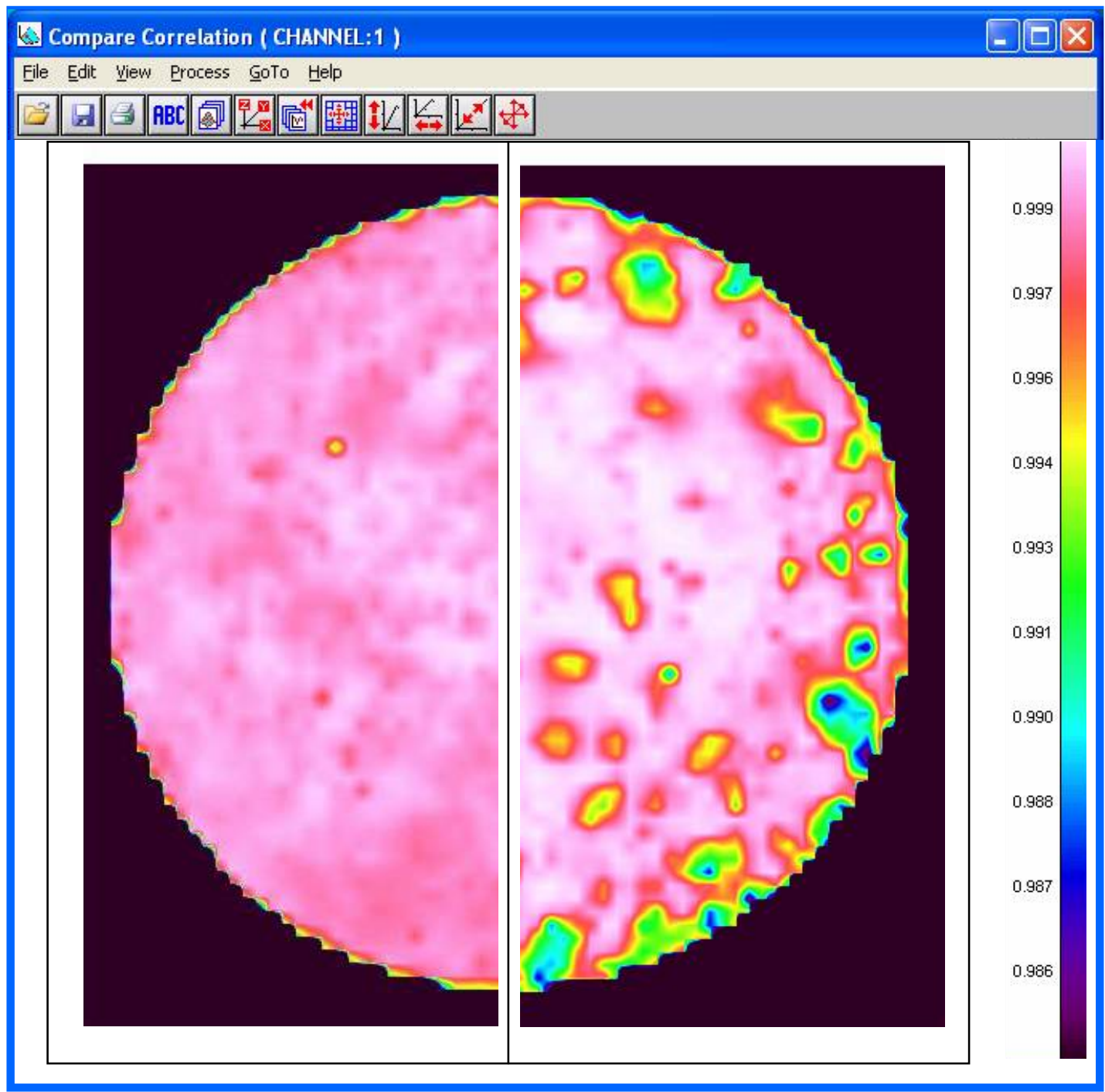

Fig. 5. Composite Raman image comparing data from two tablets with the same overall composition but different formulation protocols. The image on the right is from a tablet granulated in a single step, that on the left is from a tablet where the API was first dissolved in binder and the resulting mass reground before being granulated. In these images the positions where the spectra show the highest correlation with that that of a sample having the required API and binder/excipient band intensities are white/pink. Positions where there is poorer correlation are green/blue. (Image courtesy of Perkin Elmer Inc) 


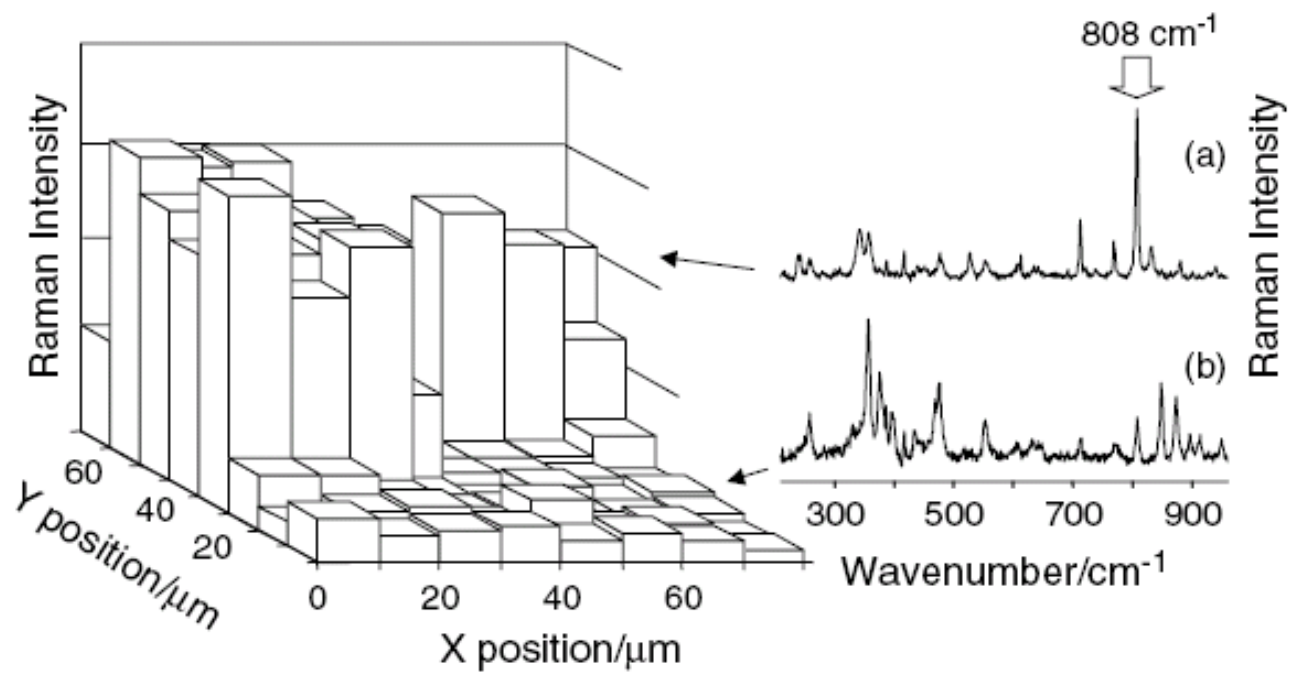

Fig. 6. Plot of the peak area of the strongest MDMA band $(12376 \mathrm{~nm})$ in the Raman spectra of a seized ecstasy tablet recorded on an $8 \times 8$ grid with $10 \mu \mathrm{m}$ spacing using a Raman microscope. The inset shows typical spectra recorded in (a) the high-drug (MDMA) region and (b) the low-drug (lactose) region [51] (Reprinted courtesy of the Journal of Raman Spectroscopy). 


\section{References}

[1] www.fda.gov/cder/OPS/PAT.htm

[2] D. Schoneker. Impact of Excipient Variability on PAT Applications: The Need for Good Qualification Processes, AAPS Annual Meeting and Exposition, October 28 November 3, 2006, San Antonio, TX.

[3] E.T. Skibsted, J.A. Westerhuis, A.K. Smilde, D.T. Witte, Examples of NIR based real time release in tablet manufacturing, J. Pharm. Biomed. Anal. 43 (2007) 1297-1305.

[4] E. Lewis, J. Schoppelrei, E. Lee, L. Kidder, Near-Infrared Chemical Imaging as a Process Analytical Tool, in: K. Bakeev (Ed), Process Analytical Technology, Blackwell Publishing, Oxford, UK, 2005, pp 187--225.

[5] G. Reich, Near-infrared spectroscopy and imaging : Basic principles and pharmaceutical applications, Adv. Drug Deliv. Rev. 57 (2005) 1109--1143.

[6] T.Herkert, H. Prinz, K.-A. Kovar, One hundred percent online indentity check of pharmaceutical products by near-infrared spectroscopy on the packaging line, Eur. J. Pharm. Biopharm. 51 (2001) 9--16.

[7] E. Räsänen, N. Sandler, Near infrared spectroscopy in the development of solid dosage forms, J. Pharm. Pharmacol. 59 (2007) 147-155. 
[8] J. Rantanen, Process analytical applications of Raman spectroscopy, J. Pharm. Pharmacol. 59(2007)171--177.

[9] P. Robert, D. Bertrand, M.F. Devaux, A. Sire, Identification of chemical constituents by multivariate near-infrared spectral imaging, Anal. Chem. 64(1992) 664--667.

[10] P. Tatzer , M. Wolf, T. Panner, Industrial application for in-line material sorting using hyperspectral imaging in the NIR range, Real-Time Imaging 11(2005) 99--107.

[11] A. Prieto, F. Bellas, F. López-Peña, R.J. Duro, Integration of Spatial Information in Hyperspectral Imaging for Real Time Quality Control in an Andalusite Processing Line, K.E.S. 3 (2006) 292--299.

[12] A. Kulcke, C. Gurschler, G. Spöck, R. Leitner, A. Kraft, On-line classification of synthetic polymers using near infrared spectral imaging, J. Near Infrared Spec. 11 (2003) 71--81.

[13] D. Ferris, R. Lawhead, E. Dickman, N. Holtzapple, J. Miller, S. Grogan, S. Bambot, A. Agrawal, M. Faupel, Multimodal hyperspectral imaging for the noninvasive diagnosis of cervical neoplasia, J. Low. Genital Tract Dis. 5(2001) 65 -72. 
[14] D. Kellicut, J. Weiswasser, S. Arora, J. Freeman, R. Lew, C. Shuman, J. Mansfield, A. Sidawy, Emerging technology: Hyperspectral imaging, Perspect. Vasc. Surg. Endovasc. Therapy 16 (2004), 53--57.

[15] G. Zheng, Y. Chen, X. Intes, B. Chance, J.D. Glickson, Contrast-enhanced nearinfrared (NIR) optical imaging for subsurface cancer detection. J. Porphyrin. Phthalocyanin. 8 (2004) 1106--1117.

[16] K.C. Lawrence, B. Park, W.R. Windham, C. Mao, Calibration of a pushbroom hyperspectral imaging system for agricultural inspection, Trans. ASAE 46 (2003) $513--521$.

[17] D. Clark, S. Šašic, Chemical images: Technical approaches and issues, Cytometry 69A (2006) 815--824.

[18] M. Henson, L. Zhang, Drug Characterization in Low Dosage Pharmaceutical Tablets Using Raman Microscopic Mapping, Appl. Spectrosc. 60 (2006) 1247--1255.

[19] K. Zuzak, M. Schaeberle, M. Gladwin, R. Cannon, I. Levin, Noninvasive Determination of Spatially Resolved and Time-Resolved Tissue Perfusion in Humans During Nitric Oxide Inhibition and Inhalation by Use of a Visible-Reflectance Hyperspectral Imaging Technique. Circulation 104 (2001) 2905--2910. 
[20] Y. Roggo, A. Edmond, P. Chalus, M. Ulmschneider, Infrared hyperspectral imaging for qualitative analysis of pharmaceutical solid forms. Anal. Chim. Acta 535 (2005) 79--87.

[21] D. Pappas, B.W. Smith, J.D. Winefordner, Raman Imaging for Two-Dimensional Chemical Analysis, Appl. Spectrosc. Rev. 35 (2000) 1--23.

[22] S. Šašic, D.A. Clark, Defining a strategy for chemical imaging of industrial pharmaceutical samples on Raman line-mapping and global illumination instruments, Appl. Spectrosc. 60 (2006) 494--502.

[23] E. N. Lewis, J. Schoppelrei, E. Lee, Near-infrared Chemical Imaging and the PAT Initiative, Spectrosc. Mag. 04 (2004) 26--36.

[24] R. Salzer, G. Steiner, H.H. Mantsch, J. Mansfield, E.N. Lewis, Infrared and Raman imaging of biological and biomimetic samples, Fresenius J. Anal. Chem. 366 (2000) 712--716.

[25] C. Pohl, Multisensor image fusion in remote sensing, Int. J. Remote Sens.19 (1998) 823--854.

[26] Y. Liu, Y.R Chen, M.S. Kim, D.E. Chan, A.M. Lefcourt, Development of simple algorithms for the detection of fecal contaminants on apples from visible/near infrared hyperspectral reflectance imaging, J. Food Eng., 81(2007) 412--418. 
[27] B. Park, K.C. Lawrence, W.R. Windham, D. Smith, Performance of hyperspectral imaging system for poultry surface fecal contaminant detection, J. Food Eng., 75 (2006) 340--348.

[28] E. N. Lewis, J. E. Carroll, F. M. Clarke, A near-infrared view of pharmaceutical formulation analysis, NIR News 12(2001) 16--18.

[29] S. Šašic, An in-depth analysis of Raman and near-infrared chemical images of common pharmaceutical tablets, Appl. Spectrosc. 61 (2007) 239--250.

[30] B.J. Westenberger, C.D. Ellison, A.S. Fussner, S. Jenney, R.E. Kolinski, T.G. Lipe, R.C. Lyon, T.W. Moore, L.K. Revelle, A.P. Smith, J.A. Spencer, K.D. Story, D.Y. Toler, A.M. Wokovich, L.F. Buhse, Quality assessment of internet pharmaceutical products using traditional and non-traditional analytical techniques, Int. J. Pharm. 306 (2005) 56-70.

[31] F. Clarke, Extracting process-related information from pharmaceutical dosage forms using near infrared microscopy, Vib. Spectrosc. 34 (2004) 25--35.

[32] Y. Roggo, N. Jent, A. Edmond, P. Chalus, M. Ulmschneider, Characterizing process effects on pharmaceutical solid forms using near-infrared spectroscopy and infrared imaging, Eur. J. Pharm. Biopharm. 61 (2005) 100--110.

[33] R.C. Lyon, D.S. Lester, E.N. Lewis, E. Lee, L.X. Yu, E.H. Jefferson, A.S. Hussain, Near-infrared spectral imaging for quality assurance of pharmaceutical 
products: analysis of tablets to assess powder blend homogeneity. AAPS PharmSciTech. 3(2002) 17.

[34] E.N. Lewis, L. Kidder, E. Lee, NIR Chemical Imaging as a Process Analytical Tool, Innov. Pharm. Tech. 17 (2006) 107--111.

[35] E. N. Lewis, L. H. Kidder, E. Lee, NIR Chemical Imaging - Near-Infrared Spectroscopy on Steroids, NIR News 16 (2005) 2.

[36] O. Rodionova, L. Houmøller, A. Pomerantsev, P. Geladi, J. Burger, V. Dorofeyev, A. Arzamastsev, NIR spectrometry for counterfeit drug detection: A feasibility study, Anal. Chim. Acta 549 (2005) 151--158.

[37] J. Dubois, J-C. Wolff, J. K. Warrack, J. Schoppelrei, E. Lewis, NIR Chemical Imaging for Counterfeit Pharmaceutical Products Analysis, Spectrosc. 22 (2007) 40-50.

[38] S.J. Hamilton, R.A. Lodder, Hyperspectral imaging technology for pharmaceutical analysis, Proc. SPIE 4626 (2002) 136-147.

[39] E. Lee, W.X. Huang, P. Chen, E.N. Lewis, R.V. Vivilecchia, High-throughput analysis of pharmaceutical tablet content uniformity by near-infrared chemical imaging, Spectrosc. 21 (2006) 24--32. 
[40] I. Malik, M. Poonacha, J. Moses, R.A. Lodder, Multispectral imaging of tablets in blister packaging, AAPS PharmSciTech (2001) 9.

[41] S.J. Hamilton, A.E. Lowell, R.A. Lodder, Hyperspectral techniques in analysis of oral dosage forms, J. Biomed. Opt. 7 (2002) 561-570.

[42] P. Taylor, Chemical imaging investigated for process monitoring, In-Pharma Technol. Newsletter Jan 2005, www.in-pharmatechnologist.com.

[43] L. Markwort, B. Kip, E. Da Silva, B. Roussel, Raman Imaging of Heterogeneous Polymers: A Comparison of Global versus Point Illumination, Appl. Spectrosc. 49 (1995) 1411--1430.

[44] D. M. Wieliczka, M. B. Kruger, P. Spencer, Raman imaging of dental adhesive diffusion, Appl. Spectrosc. 51 (1997) 1593--1596.

[45] X. M. Yang, D. A. Tryk, K. Hasimoto, A. Fujishima, Surface enhanced Raman imaging of a patterned self-assembled monolayer formed by microcontact printing on a silver film, Appl. Phys. Lett. 69 (1996) 4020--4022.

[46] M. Escoriza, J. VanBriesen, S. Stewart, J. Maier, P. Treado, Raman spectroscopy and chemical imaging for quantification of filtered waterborne bacteria, J. Microbiol. Meth. 66 (2006) 63--72. 
[47] J. Ling, S.D. Weitman, M.A. Miller, Methodology of using raman imaging microscopy for evaluating drug action within living cells. U.S. Patent 6,939,686 (2005).

[48] W.H. Doub, W.P. Adams, J.A. Spencer, L.F. Buhse, M.P. Nelson, P.J. Treado, Raman chemical imaging for ingredient-specific particle size characterization of aqueous suspension nasal spray formulations: a progress report. Pharm. Res. 24 (2007) 934--945 .

[49] S. Šašic, Raman mapping of low-content API pharmaceutical formulations. I. Mapping of alprazolam in Alprazolam/Xanax tablets, Pharm. Res. 24 (2007) 58--65.

[50] E. Lee, F. Adar, A. Whitley, The impact of spatial sampling density in Raman imaging, Spectrosc 21S (2006) 21.

[51] S.E.J. Bell, J.R. Beattie, J.J. McGarvey, K.L. Peters, N.M.S. Sirimuthu, S.J. Speers, Development of sampling methods for Raman analysis of solid dosage forms of therapeutic and illicit drugs, J. Raman Spectrosc. 35 (2004) 409--417.

[52] A. De Juan, R. Tauler, R. Dyson, C. Marcolli, M. Rault, M. Maeder, Spectroscopic imaging and chemometrics: a powerful combination for global and local sample analysis, Trend. Anal. Chem. 23 (2004) 70--79. 
[53] S. Šašic, D.A. Clark, J.C. Mitchell, M.J. Snowden, Analyzing Raman maps of pharmaceutical products by sample-sample two-dimensional correlation, Appl. Spectrosc. 59 (2005) 630--638.

[54] L. Zhang, M.J. Henson, S.S. Sekulic, Multivariate data analysis for Raman. imaging of a model pharmaceutical tablet, Anal Chim Acta 545 (2005) 262--278.

[55] S.E.J. Bell, L.J. Barrett, D.T. Burns, A.C. Dennis, S.J. Speers, Tracking the distribution of "ecstasy" tablets by Raman composition profiling: a large scale feasibility study, Analyst 128 (2003) 1331--1335.

[56] S.E.J. Bell, D.T. Burns, A.C. Dennis, J.S. Speers, Rapid analysis of ecstasy and related phenethylamines in seized tablets by Raman spectroscopy, Analyst 125 (2000) $541--544$.

[57] S.E.J. Bell, D.T. Burns, A.C. Dennis, Matchett LJ, Speers JS, Composition profiling of seized ecstasy tablets by Raman spectroscopy, Analyst 125 (2000) 1811-1815.

[58] C.A. Shah, P. Watanachaturaporn, P.K. Varshney, M.K. Arora, Some recent results on hyperspectral image classification, In Proceedings of IEEE Workshop on Advances in Techniques for Analysis of Remotely Sensed Data (pp. 346--353), Greenbelt (MD), 27-28 October 2003. 
[59] H. Noh, R. Lu, Hyperspectral laser-induced fluorescence imaging for assessing apple fruit quality, Postharvest Biol. Tec. 43 (2007)193--201.

[60] R.P. Cogdill, P. Dardenne, Least-squares support vector machines for chemometrics: an introduction and evaluation, J. Near Infrared Spec. 12 (2004) 93-100.

[61] L. Jiang, B. Zhu, X. Rao, G. Berney, Y. Tao, Discrimination of black walnut shell and pulp in hyperspectral fluorescence imagery using Gaussian kernel function approach, J. Food Eng. 81(2007) 108-117.

[62] S. Schlucker, M.D. Schaeberle, S.W. Huffman, I.W. Levin, Raman microspectroscopy: a comparison of point, line, and wide-field imaging methodologies, Anal. Chem. 75 (2003) 4312--4318.

[63] F. Koehler, E. Lee, L. Kidder, N. Lewis. Near infrared spectroscopy: the practical chemical imaging solution, Spectroscopy Eur. 14(2002)12--19.

[64] Chen, Y.R., Chao, K., \& Kim, M.S. (2002). Machine vision technology for agricultural applications. Computers and Electronics in Agriculture, 36(2), 173--191.

[65] F.C. Clarke, M.J. Jamieson, D.A. Clark, S.V. Hammond, R.D. Jee, A.C. Moffat, Chemical Image Fusion. The Synergy of FT-NIR and Raman Mapping Microscopy To Enable a More Complete Visualization of Pharmaceutical Formulations, Anal. Chem. 73 (2001) 2213--2220. 
[66] L.A. Cassis, A. Urbas, R.A Lodder, Hyperspectral integrated computational imaging, Anal. Bioanal. Chem. 382 (2005) 868--872.

[67] J. Medendorp, R.A. Lodder, Applications of integrated sensing and processing in spectroscopic imaging and sensing, J. Chemometr. 19 (2005) 533--542.

[68] V. Bellon-Maurel, NIR imaging spectroscopy instrumentation: looking forward to 2020?, NIR news, 17 (2006)14--23. 